\title{
¿Cuán abierta es la producción científica de los investigadores argentinos de Ciencias Sociales?
}

How open is the scientific production of Argentine Social Sciences researchers?

Paola C. Bongiovani

Universidad Nacional de Rosario.

Facultad de Humanidades y Artes, Argentina

Sandra E. Miguel

Instituto de Investigaciones en Humanidades y Ciencias Sociales-IdIHCS (UNLP-CONICET).

Universidad Nacional de la Plata, Argentina

\section{Resumen:}

Este estudio exploratorio se propuso conocer la disponibilidad en abierto de la producción científica argentina de Ciencias Sociales incluida en Scopus en 2017, estimar el costo por la publicación en revistas de acceso abierto e híbridas, que cobran cargos de APCs y establecer si existe una correlación entre el precio de APC (article processing charges) de las revistas y su impacto. Los resultados indican que de un total de 1480 documentos el 61 \% (907) están disponibles en Acceso Abierto, siendo este porcentaje mucho más alto que el promedio a nivel mundial. De este grupo, el $95 \%$ (864) fue publicado en revistas de acceso abierto que no cobran APCs. Con relación al valor de impacto SJR (Scimago Journal Rank) se obtuvo que las revistas híbridas están mayormente ubicadas en el cuartil Q1 y Q2, mientras que las revistas AA están distribuidas en todos los cuartiles, con mayor concentración en Q2 y Q3. Se concluye que, pese a que es alto el porcentaje de documentos disponibles en abierto sin pago de APCs, lograr el acceso abierto siguiendo los principios del Plan $S$ requeriría a los organismos financiadores argentinos asumir costos importantes con una estimación por año de U\$D 897.222, solo para la producción con visibilidad en Scopus del área de Ciencias Sociales.

Palabras clave: Acceso abierto, Producción científica, Modelos de acceso, Costo de publicación, Ciencias Sociales, Argentina.

\section{Abstract:}

This exploratory study analyzes the Open Access (OA) availability of the Argentine Social Science scientific production included in Scopus in 2017, estimates the cost for OA and hybrid journals and establishes if there is a correlation between journal's APCs price and their impact. The results indicate that of a total of 1480 documents, $61 \%$ (907) are available in OA, this percentage is higher than the global average. Of this group, 95\% (864) was published in OA journals that do not charge APCs. Regarding impact value SJR (Scimago Journal Rank) it was obtained that hybrid journals are mostly located in quartile Q1 and Q2, while AA journals are distributed in all quartiles, with greater concentration in $\mathrm{Q} 2$ and $\mathrm{Q} 3$. The conclusion of this study is that although the percentage of documents available OA without payment of APCs is high, achieving open access following the principles of Plan S would require Argentine financing agencies to assume significant costs with an estimate of US $\$ 897,222$ per year, for Social Sciences outputs visible in Scopus.

KeYwords: Open Access, Scientific production, Access models, Publication costs, Social sciences, Argentina.

\section{INTRODUCCIÓN}

Tradicionalmente para las revistas internacionales denominadas de "corriente principal”, el modo de acceso a la literatura científica publicada en revistas estuvo basado en el modelo de suscripción. Es decir, los investigadores enviaban sus manuscritos para publicación (en algunos casos pagando un canon), cedían a las editoriales los derechos de reproducción de sus artículos y los lectores pagaban por su acceso, ya sea por medio de suscripciones individuales o institucionales. 
El movimiento de acceso abierto (AA), impulsado a partir de las declaraciones de Budapest Open Access Initiative (2002, 2012); la Declaración de Bethesda (2003), la Declaración de Berlín (2003) sobre publicaciones de Humanidades y Ciencias Sociales y la Declaración de Salvador, Brasil (2005) (International Seminar on Open Access, 2006), propuso transformar este modelo con un doble fin: garantizar a los lectores la disponibilidad gratuita a la literatura científica y abolir la cesión de derechos de reproducción a las editoriales, para que cualquier usuario pueda leer, descargar, copiar, distribuir, imprimir, buscar o añadir un enlace al texto completo de esos artículos, rastrearlos para su indización, incorporarlos como datos en un software, o utilizarlos para cualquier otro propósito que sea legal, sin barreras financieras, legales o técnicas, aparte de las que son inseparables del acceso mismo a la Internet. Para alcanzar estos objetivos se propusieron originalmente dos vías: la dorada y la verde. Mientras la primera, la más purista, requería que las revistas adopten el modelo de acceso abierto, la segunda dejaba la liberación del acceso en manos de los autores, requiriendo el depósito de una copia de sus artículos en repositorios de acceso abierto, previo consentimiento/permiso otorgado por los editores.

El modelo de gestión y acceso seguido por muchas revistas en América Latina, sostenidas y financiadas principalmente por las universidades, ha estado desde sus inicios basado en la cooperación donde no pagan ni los lectores ni los autores. Así surgieron portales de revistas regionales como SciELO (1997) y RedALyC (2002) entre otras iniciativas de la región que promueven el acceso abierto desde su surgimiento.

A nivel europeo y latinoamericano existen dos iniciativas recientes, Plan $S$ y AmeliCA, que se proponen tener un importante efecto en el modelo de acceso a las revistas. Por un lado, el Plan S (Science Europe, 2018) cuenta con el compromiso de los organismos financiadores más importantes de Europa y se hará efectivo en 2021. Este plan contiene diez principios fundamentales entre los cuales se indica que los autores y sus instituciones retendrán el copyright de sus publicaciones sin restricciones y sin periodos de embargo y que no se financiarán las publicaciones en revistas híbridas a menos que estén en proceso de transformación hacia revistas de acceso abierto. Si bien el Plan $S$ se compromete a sostener una variedad de modalidades de acceso, indica que los financiadores de investigación permitirán el pago de APCs "acordes con los servicios de publicación ofrecidos y la estructura de dichas tarifas debe ser transparente para informar al mercado y a los financiadores la posible estandarización y el límite de los pagos de las tarifas" (Science Europe, 2018).

AmeliCA, por su parte, plantea una infraestructura de comunicación para la publicación académica y la ciencia abierta. Es una iniciativa puesta en marcha por instituciones latinoamericanas con objetivos comunes, planteando "estrategias, en respuesta al contexto internacional, regional, nacional e institucional, que busca una solución de Acceso Abierto colaborativa, sostenible, protegida y no comercial para América Latina y el Sur Global" (Becerril-García et al., 2018). AmeliCA, a diferencia del Plan S, considera que los autores no deben pagar por las publicaciones y propone la protección legal del acceso abierto con licencias que no permitan los usos comerciales de obras. Asimismo, AmeliCA intenta que las instituciones y gobiernos latinoamericanos financiadores de la ciencia en la región no se adhieran al Plan $S$.

Sin embargo, el Ministerio de Educación, Cultura, Ciencia y Tecnología de la Argentina firmó junto con la Unión Europea el "JOINT COMMUNIQUÉ. XI Joint Steering Committee Meeting of the Bilateral Agreement on Science and Technology between the European Union and Argentina” en junio de 2019, en el cual se indica que Argentina se unirá a Coalition $S$ y promoverá esta iniciativa regional entre los países de América Latina y el Caribe (Argentina-Unión Europea, 2019). No hay información aún sobre presupuestos destinados específicamente a los pagos de APCs que debería afrontar Argentina.

Paralelamente a las negociaciones en torno a la adopción de modelos de publicación y de acceso, y la convergencia de iniciativas que plantean diferentes estrategias en relación a los aspectos de pago y la protección legal de las producciones, se vienen realizando diversos estudios sobre el impacto del acceso abierto en la comunicación científica. Algunos trabajos analizan el éxito (o no) del AA en términos de la cantidad de literatura disponible en abierto en la web. Las cifras más recientes revelan que cerca de un $25 \%$ de los artículos de investigación recientes son accesibles por la vía dorada, y un 15-20 \% por la verde (Björk, 2017b). 
Por su parte Piwowar et al. (2018) y Piwowar, Priem y Orr (2019) estimaron que la literatura científica en acceso abierto alcanzó al $28 \%$ en 2018, el $31 \%$ en 2019 y que para 2025 se llegará a un $44 \%$. Otros estudios enfocan la atención en los efectos de la iniciativa en los modelos de publicación de las revistas, especialmente de las grandes editoriales comerciales, y en la diversificación de las vías tradicionales del AA. Fuchs y Sandoval (2013) proponen la denominación de vía diamante para referir al modelo de publicación y acceso gratuito para autores y lectores, pero con restricciones a la reutilización comercial de los artículos. Piwowar et al. (2018) denominan vía bronce a las revistas que no tienen políticas explícitas de licencia abierta pero sus artículos son de lectura libre y gratuita en sus sitios web. Por otro lado, existe el modelo híbrido, en crecimiento, que comprende a las revistas tradicionales de suscripción que ofrecen a los autores la posibilidad de pagar un cargo por publicación (APC, article processing charge) para que sus trabajos estén disponibles en acceso abierto en la web (Björk, 2012 y 2017a). A lo que se agregan formas no legales que Björk (2017b) llama vía negra, especialmente representada por la disponibilidad del texto completo de artículos en plataformas como ResearchGate y Sci Hub.

Exceptuando la vía negra, y las revistas de suscripción que ofrecen algún permiso de autoarchivo, conocer qué modelo de acceso abierto adopta cada revista no siempre resulta claro. Por ejemplo, el directorio DOAJ (Directory Open Access Journal) ofrece para cada revista que indiza información sobre permisos de reproducción y uso (licencia), así como si cobra APC. En otros casos, como el de las revistas indizadas en la base de datos Scopus, solo existe la etiqueta "Open Access" sin distinción del modelo de AA adoptado.

Algunos estudios recientes analizan la relación del cobro por APC e indicadores de impacto. Björk y Solomon (2015) encontraron una correlación positiva moderada entre los precios de revistas AA cobrados por APC y los valores de SNIP (Source Normalized Impact per Paper), una medida de calidad de las revistas que tiene en cuenta las citas totales recibidas por las revistas en un área determinada. Por otro lado, en el Reino Unido se encontró que los costos de APCs de las revistas híbridas son mucho más altos que los de las revistas AA (Pinfield, Salter y Bath, 2016 y 2017), pero también se encontró una correlación positiva entre este costo y una medida de calidad, el Field Weighted Citation Index (FWCI) de Scopus. Se observó además que el cambio político donde se enfatizó el pago de APCs por parte de las instituciones en el Reino Unido significó que un 12 \% del costo total de las publicaciones fue destinado al pago de APCs entre 2012 y 2014 (Pinfield et al., 2017).

En otra investigación Khoo (2019) señala que los autores no parecen considerar el precio del APC al momento de publicar sus artículos, ya que si no pueden pagarlo optan por no disponer en abierto sus trabajos, es decir, que el acceso es por suscripción. A menos que los financiadores hagan valer su poder de negociación y establezcan políticas para limitar los costos, la insensibilidad de los autores a los precios hará que el acceso abierto financiado por APCs dé continuidad a la crisis de las revistas, origen del movimiento de AA.

\section{El CASO ARgentino}

En Argentina, Miguel, Gómez y Bongiovani (2012) analizaron el acceso abierto real y potencial de la producción científica argentina publicada entre 2008 y 2010 en revistas indizadas por Scopus. Encontraron que el $25 \%$ de la producción había sido publicada en revistas de la tradicional vía dorada, aunque había variantes segn las áreas temáticas: Agricultura y Ciencias Biológicas (22,6\%), Física y Astronomía (9,1 \%), Medicina (33,7 \%) y Ciencias Sociales (43,1\%). Para las mismas áreas, otro estudio mostró que un alto porcentaje de los investigadores argentinos expresan conocer la existencia de revistas de acceso abierto en sus campos del conocimiento, y que lo consideran beneficioso principalmente por el bien de la comunidad científica, por razones financieras y para el bien públicoSin embargo, el prestigio de las revistas es el factor preponderante a la hora de elegir dónde publicar, y la baja calidad de las revistas y los costos por publicación aparecen como dos factores que creen desfavorables (Bongiovani, Gómez y Miguel, 2012). 
En un estudio más reciente, Bongiovani, Miguel y Hernández-Pérez (2017) encontraron que los evaluadores de la carrera científica en Argentina mantienen opiniones y actitudes tradicionales, privilegiando la revisión por pares, el prestigio de la revista, el factor de impacto y la indización en índices internacionales y regionales. El modelo de acceso abierto de la revista no aparece como factor clave ni en la publicación ni en la evaluación de los investigadores. Un dato revelador que contrasta en parte estas opiniones es que el $61 \%$ de los evaluadores había publicado al menos un artículo en revistas AA en los últimos cinco años.

\section{OBjetivos}

En este escenario, y a partir de los resultados previos que muestran que el área de Ciencias Sociales es en la producción científica argentina con visibilidad internacional en Scopus la que registra más porcentaje de trabajos publicados en AA, y que no se encontraron investigaciones en el área sobre el modelo de acceso abierto en relación al costo por APC de las revistas donde publican los investigadores argentinos, este trabajo se propone como objetivos:

- Conocer la disponibilidad en abierto de la producción científica argentina de Ciencias Sociales incluida en Scopus.

- Estimar cuál es el costo por la publicación en revistas de acceso abierto e híbridas, que cobran cargos de APC.

- Establecer si existe relación entre el precio de APC de las revistas y una medida de impacto, el SJR (Scimago Journal Rank).

En estudios posteriores se analizarán y realizarán comparaciones con las revistas y la producción en otras áreas temáticas.

\section{Metodología}

Se considera que la producción científica argentina de Ciencias Sociales es amplia y se publica de formas muy diversas, lo cual limita su análisis completo. Para los objetivos de carácter exploratorio del estudio, solo se consideró la producción científica argentina compuesta por artículos de revistas y demás tipos documentales de Ciencias Sociales y del año 2017, recogida de la base de datos SCOPUS, de Elsevier, el 8 de abril de 2019. Los registros seleccionados tenían la mención de una institución argentina en los datos de afiliación institucional de al menos uno de los autores firmantes del artículo, y se filtró por área temática Ciencias Sociales y año de publicación. En total se exportaron 1480 registros.

Para establecer el porcentaje de la producción científica argentina de 2017 en Ciencias Sociales que se encuentra disponible en acceso abierto se utilizó la herramienta oaDOI. Esta es un servicio de Unpaywall que se inició en 2016 por Heather Piwowar y Jason Priem para identificar si existe una versión en acceso abierto de documentos identificados por su DOI (Digital Object Identifier). El objetivo es brindar el estatus de los artículos en acceso abierto, incluyendo la licencia de acceso ya sea a través de los editores, abarcando revistas híbridas y repositorios.

Los documentos con DOI que pudieron analizarse a través de este servicio suman 1107 (75 \% de la producción). Los resultados de oaDOI brindan información de cómo se encontró la evidencia de acceso abierto. Es importante considerar que las categorías que esta base de datos utiliza como evidencia de acceso abierto son excluyentes y el servicio oaDOI privilegia resultados de las editoriales y sitios web de las revistas; si no están allí, ofrece la evidencia de acceso en un repositorio de acceso abierto. Esta limitación no permite saber qué porcentaje total de la producción encontrada en acceso abierto está en repositorios. 
Para determinar el modo de acceso al total de la producción publicada en 2017 (1480 documentos), y el costo del APC a los documentos publicados en revistas híbridas, se verificaron los datos de las revistas en las siguientes bases de datos y sitios:

- DOAJ (Directory of Open Access Journals)

- SciELO (Scientific Electronic Library Online)

- Redalyc

- Sitios de las editoriales

- Sitios web de las revistas, conferencias y libros

La mayoría de los precios de APCs de las revistas estaban en dólares estadounidenses (USD). En los pocos casos en que aparecían en otras monedas, los precios de APCs fueron convertidos a USD utilizando la herramienta XE Currency Converter (https://www.xe.com/).

También se chequeó el sitio Scimago Journal \& Country Rank (2019) para ver el impacto asignado a las revistas a partir del indicador SJR, que se obtiene a partir del cálculo de las citas recibidas por las revistas en un periodo de 3 años, otorgando un peso mayor a las citas procedentes de revistas de alto prestigio (aquellas con altas tasas de citación y baja autocitación) utilizando para ello el algoritmo de Google PageRank.

\section{RESULTADOS Y DISCUSIÓN}

\section{Documentos en acceso abierto según oaDOI}

El servicio oaDOI encontró que el 52,6\% de los 1107 documentos analizados están en acceso abierto, ya sea a travs de revistas AA (45,3\%), repositorios (5,2 \%), revistas híbridas $(1,4 \%)$ o revistas bronce $(0,6 \%)$. El resto de los documentos $(47,4 \%)$ se encuentran en revistas, actas de conferencias o libros cerrados (por suscripción) (figura 1). Sin embargo, es posible que existan más documentos en acceso abierto a travésle la vía verde (repositorios) ya que oaDOI no captura este dato si encuentra la versión de la editorial.

Figura 1: Porcentaje de documentos por categoría de acceso. $\mathrm{N}=1.107$

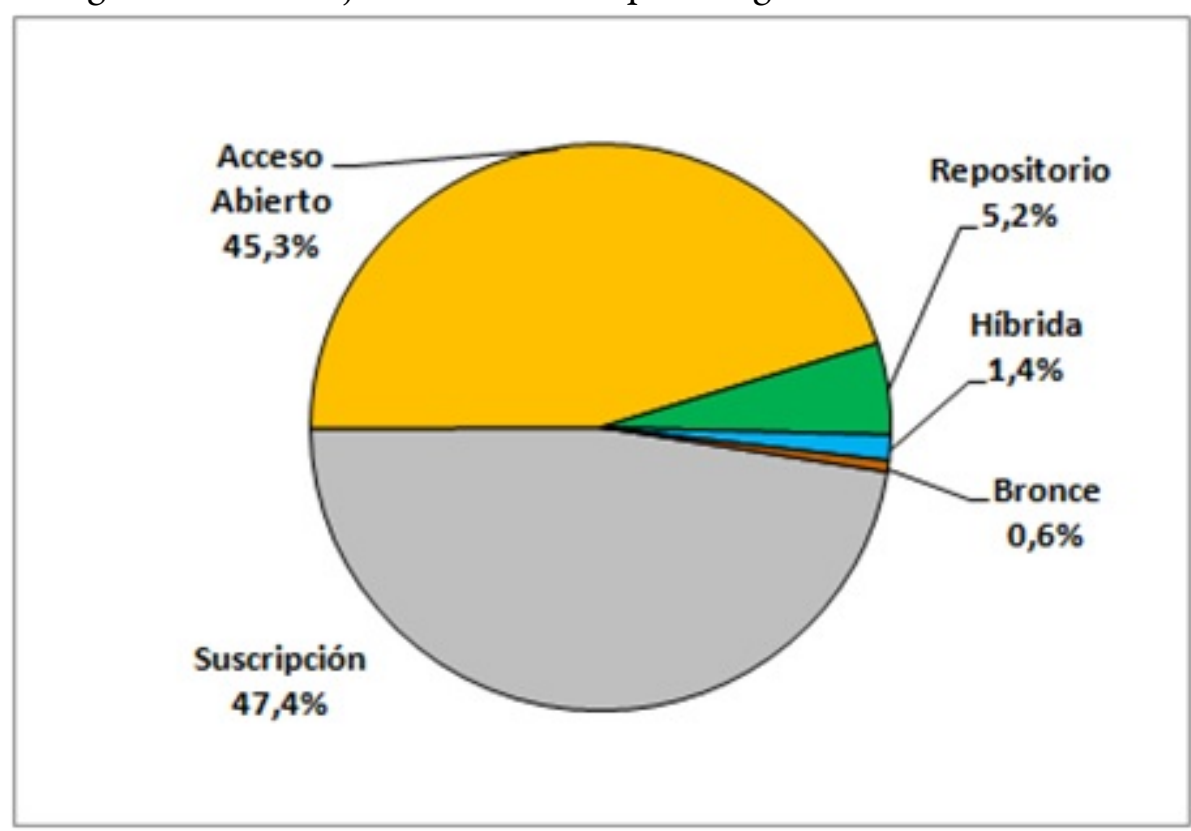

Fuente: Elaboración propia 
Al verificar cada uno de los documentos en la muestra, es decir aquellos con DOI o sin DOI, en DOAJ, SciELO, RedALyC, sitios de las editoriales y revistas, se encontró que los 1480 documentos fueron publicados en 670 fuentes, principalmente revistas científicas, pero también en actas de conferencia, libros y capítulos de libro. Al revisar las fuentes mencionadas se identificó que un $61 \%$ de los documentos fueron publicados en revistas de acceso abierto, un $21 \%$ en revistas híbridas, un $17 \%$ en fuentes por suscripción. Se encontraron 6 documentos publicados en revistas impresas y 1 que estaba en acceso abierto pero la revista lo difunde luego de 12 meses de embargo (figura 2).

Figura 2: Porcentaje de documentos por categoría de acceso. $\mathrm{N}=1.480$

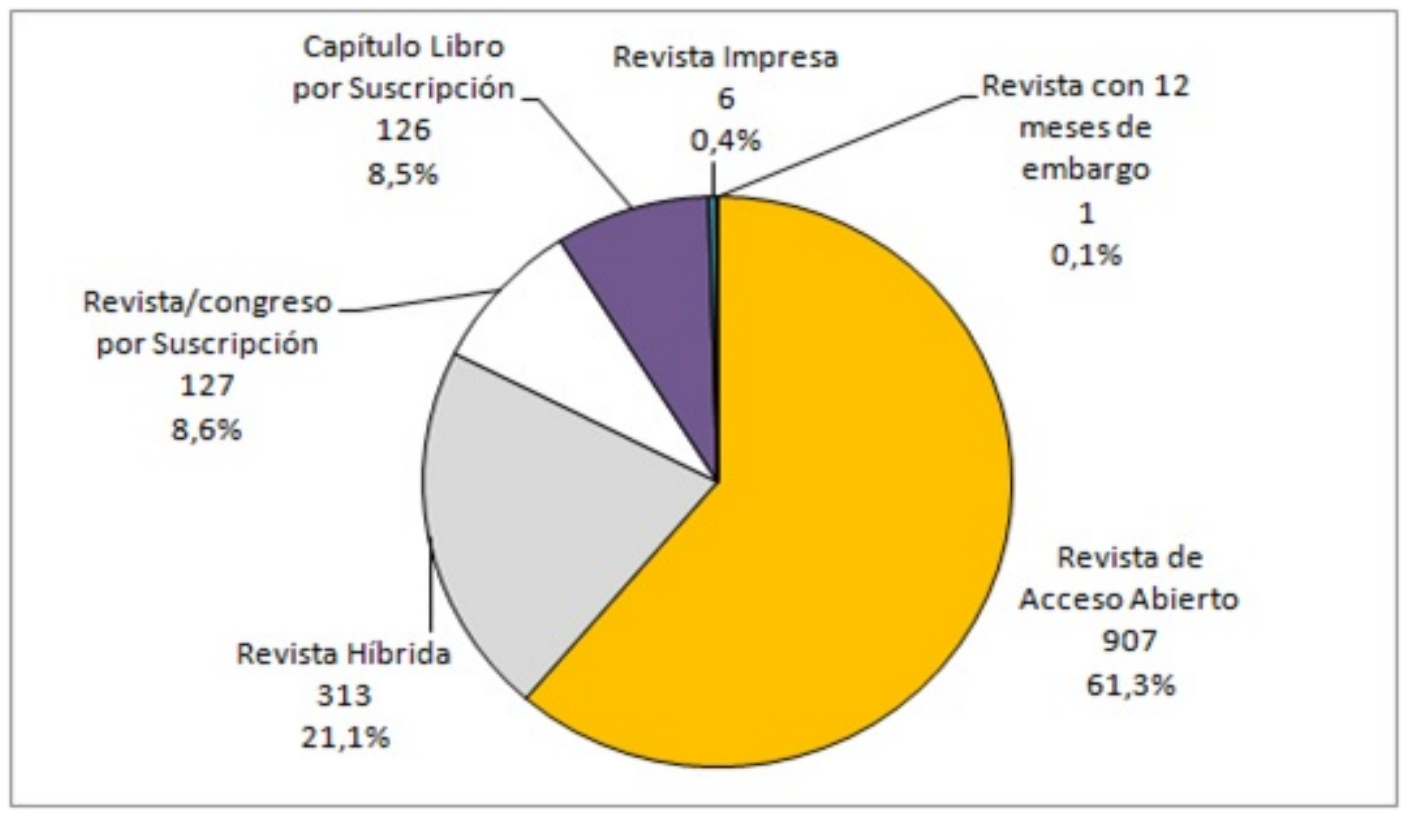

Fuente: Elaboración propia.

El mayor porcentaje de artículos en revistas de acceso abierto encontrados en este análisis exhaustivo, se debe principalmente a que hay muchas revistas latinoamericanas AA que aún no asignan DOIs a sus artículos, y por esa razón no fueron incluidas en el análisis con el oaDOI.

Si se comparan estos resultados con la investigación publicada en 2012 (Miguel et al., 2012) que abarcaba artículos del área de Ciencias Sociales publicados por investigadores argentinos en el periodo 2008-2010, se observa un aumento en la disponibilidad de artículos en acceso abierto del $43 \%$ al $61 \%$.

\section{Costo del acceso abierto}

De los 907 documentos publicados en acceso abierto en 2017, la gran mayoría (864) fue publicado en revistas AA que no cobran APC. Esto se relaciona con la tradición latinoamericana de publicaciones sostenidas por la academia y portales regionales de acceso abierto no comercial. Solamente 43 revistas cobran APC, por un total de U\$D 44.433, con un promedio de U\$D 1.033 pagado por artículo. El rango de precios varía entre U\$D 67 y U\$D 3900 por artículo. No se pudo contar con datos para identificar si este costo fue pagado por investigadores argentinos o por colegas de otros países que firmaron en coautoría.

Asimismo, se analizó el costo del APC de los artículos que se publicaron en 313 revistas híbridas cuyo valor ascendería a U\$D 852.789, si se quisiera su disponibilidad en acceso abierto en el sitio de la revista. El rango de precios del APC en este caso varía entre U\$D 1.000 y U\$D 4.200, siendo el costo promedio por artículo de U\$D 2.725. En 4 revistas no se pudo encontrar el precio del APC ni en el sitio web de la revista ni en el de la editorial. De este grupo de revistas híbridas, solamente 16 artículos se encuentran efectivamente 
publicados en acceso abierto con un costo de U\$D 39.845. Nuevamente no se puede saber si este costo fue asumido por los investigadores argentinos o sus colegas extranjeros.

\section{Impacto}

Del total de documentos, 1214 fueron publicados en revistas y conferencias que tienen indicador de impacto SJR (Scimago Journal Rank) y están segmentadas en cuartiles en el portal de SCImago. En la figura 3, se observa que las revistas híbridas están mayormente ubicadas en el cuartil Q1 y Q2, mientras que las revistas AA están distribuidas en todos los cuartiles, con mayor concentración en Q2 y Q3.

Figura 3: Distribución de las revistas de acceso abierto e híbridas de acuerdo al ranking SJR

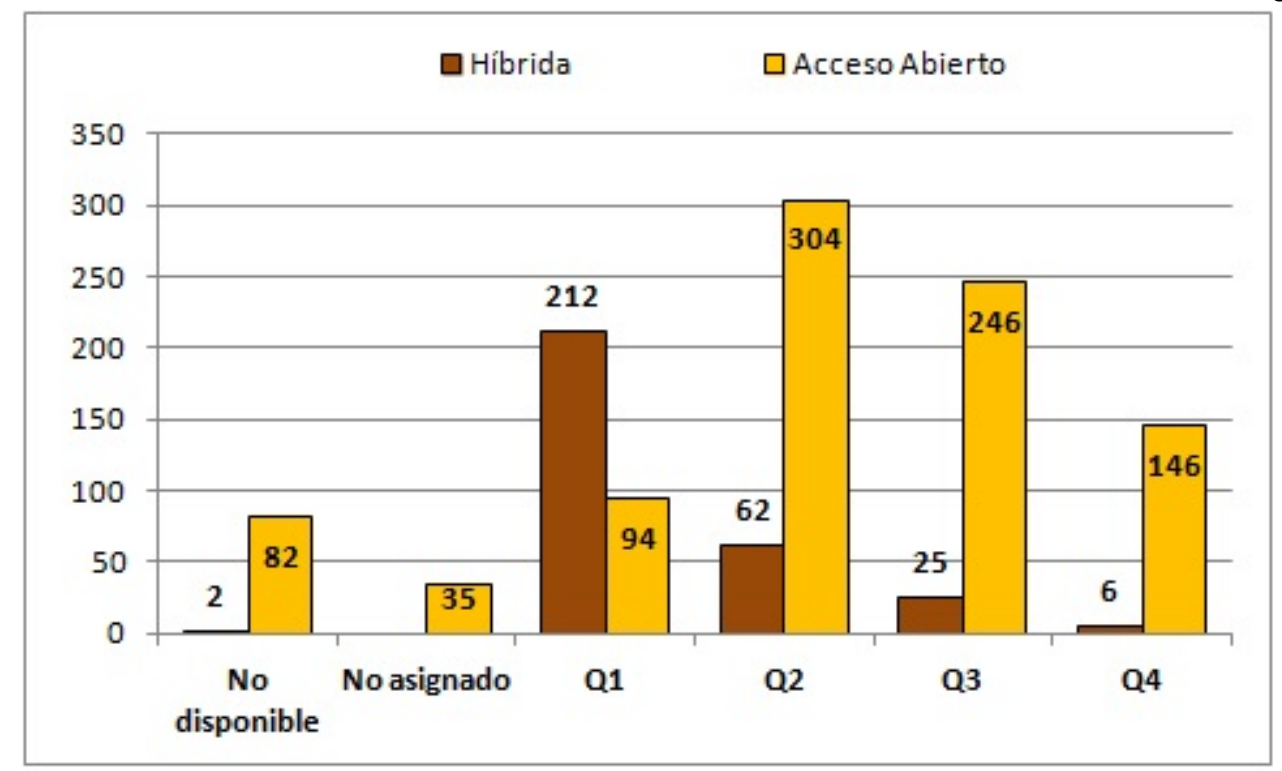

\section{Conclusiones}

Encontramos que un alto porcentaje de documentos en Ciencias Sociales publicados por investigadores argentinos en 2017 en fuentes indizadas en Scopus está disponible en acceso abierto. Segn el servicio de oaDOI el 52,6\% de los documentos están efectivamente disponibles en abierto en la web. Al realizar un análisis más exhaustivo, incluyendo a las revistas sin DOIs este porcentaje asciende a $61,3 \%$, la mayoría procedentes de Latinoamrica. Este valor indica un crecimiento de la disponibilidad en acceso abierto de artículos en Ciencias Sociales respecto de los hallazgos para el período 2008-2010 (Miguel et al., 2012), lo cual podría deberse a un incremento de las revistas AA latinoamericanas en la fuente de datos. Asimismo, este porcentaje es mucho mayor que lo observado a nivel mundial por Piwowar et al. (2018) donde la disponibilidad en acceso abierto de artículos en Ciencias Sociales no llega al $30 \%$.

Se evidencia que las instituciones educativas latinoamericanas son las principales proveedoras de publicaciones de acceso abierto sin costo para los lectores ni autores, disponibilizadas en plataformas regionales como SciELO y RedALyC. Por lo que sus políticas en relación al AA estarían más en consonancia con la iniciativa AmeliCA, que surge desde RedALyC, que con el plan S.

Si los organismos financiadores argentinos quisieran seguir los principios del Plan $S$ deberían asumir costos importantes, estimado en U\$D 897.222 por año, solo para la producción con visibilidad en Scopus del área de Ciencias Sociales. Este cálculo suma el costo de APCs de las 43 revistas de AA (U\$D 44.433) más el 
potencial costo de APCs de los 313 artículos publicados en revistas híbridas (U\$D 852.789). También, y en coincidencia con estudios previos, los resultados muestran que el costo promedio de APC por publicar en revistas de acceso abierto es menor al costo por publicar en revistas híbridas. Por otra parte, lo que cuesta sostener una publicación de acceso abierto no comercial en Argentina, es ostensiblemente menor, alcanzando una cifra aproximada a los U\$D 135 por artículo (Banzato y Rozemblum, 2019). Es evidente que los costos impuestos por las grandes editoriales son exageradamente superiores a los estimados por publicar en revistas latinoamericanas de acceso abierto.

Identificar estos costos es muy relevante, teniendo en cuenta la comunicación reciente del "Comité directivo sobre cooperación en ciencia y tecnología entre la Unión Europea y Argentina" que anuncia que Argentina se unirá a la cOAlition $S$, hecho que todavía no se ha plasmado en políticas concretas, y que requiere una respuesta cautelosa a nivel regional. La implementación del Plan $S$ podría alentar un cambio en las revistas académicas a modelos de publicación que cobran cargos por procesamiento de artículos (APC), con el peligro de ocasionar una disminución de recursos en infraestructura pública no comercial de comunicaciones científicas abiertas esenciales para avanzar hacia procesos y prácticas de ciencia abierta en América Latina (Debat y Babini, 2019).

Que el porcentaje de artículos publicados en revistas híbridas sea de apenas un $21 \%$ respecto del $61 \%$ publicado en revistas AA, confirma que el elevado costo por APC es un factor desfavorable para el acceso abierto, tal como fuera señalado por los investigadores argentinos en un estudio anterior (Bongiovani et al., 2012). Este hecho es preocupante, ya que, si los autores no pueden asumir los costos de APC para el acceso abierto, y siguen siendo evaluados con criterios tradicionales, de acuerdo con el impacto que tienen sus publicaciones (Bongiovani et al., 2017), publicarán sus trabajos en revistas por suscripción (Khoo, 2019).

Por otro lado, el prestigio de las revistas, considerado uno de los factores más importantes por los investigadores argentinos a la hora de elegir dónde publicar, se vería afectado, si se tiene en cuenta la relación del impacto medido con el indicador SJR y la categoría de acceso, que favorece más a las revistas híbridas (que se concentran en los cuartiles Q1 y Q2) que a las de acceso abierto. En este sentido se observa cierta similitud con los resultados encontrados por Björk y Solomon (2015) y Pinfield et al. (2016 y 2017) que hallaron que las revistas que cobran APC alcanzarían valores de impacto más elevados.

A modo de conclusión final, resulta evidente que alcanzar el acceso abierto mediante pago por publicación requiere asumir costos que al menos hasta ahora no están siendo contemplados en el financiamiento a la investigación en el sistema científico argentino, y refuerzan la idea de solventar las propuestas del Plan $S$ tiene implicancias económicas que habría que considerar cuidadosamente.

\section{REFERENCIAS}

Argentina-Unión Europea (2019).Joint Communiqué. XI Joint Steering Committee Meeting of the Bilateral Agreement on Science and Technology between the European Union and Argentina. Recuperado de http://ec.europa.eu/rese $\mathrm{arch} / \mathrm{iscp} / \mathrm{pdf} /$ policy/ec_rtd_jc-11th-jscm-euar_062019.pdf

Banzato, G., y Rozemblum, C. (2019). Modelo sustentable de gestión editorial en Acceso Abierto en instituciones académicas: Principios y procedimientos. Palabra Clave, 8(2). https://doi.org/10.24215/18539912e069

Becerril-García, A., Aguado-López, E., Batthyány, K., Melero, R., Beigel, F., Vélez Cuartas, G., ... y Torres, J. (2018). AmeliCA: Una estructura sostenible e impulsada por la comunidad para el Conocimiento Abierto en América Latina y el Sur Global. México: Redalyc; Universidad Autónoma del Estado de México; Argentina: CLACSO; Universidad Nacional de La Plata; Colombia: Universidad de Antioquia. Recuperado de http://www.memori a.fahce.unlp.edu.ar/libros/pm.693/pm.693.pdf

Berlin Declaration on open access to knowledge in the sciences and humanities (2003). Recuperado de http://www.zim .mpg.de/openaccess-berlin/berlindeclaration.htm 
Bethesda Statement on Open Access Publishing (2003). Recuperado de https://legacy.earlham.edu/ peters/fos/bet hesda.htm

Björk, B. (2012). The hybrid model for open access publication of scholarly articles—a failed experiment? Journal of the American Society of Information Sciences and Technology, 63(8), 1496-1504. https://doi.org/10.1002/asi.22709

Björk, B. (2017a). Gold, green and black Open Access. The Association of Learned \& Profesional Society Publishing, 30, 173-175. https://doi.org/10.1002/leap.1096

Björk, B. (2017b). Growth of hybrid open access, 2009-2016. PeerJ, 5, e3878. https://doi.org/10.7717/peerj.3878

Björk, B. C., y Solomon, D. (2015). Article processing charges in OA journals: relationship between price and quality. Scientometrics, 103(2), 373-385. https://doi.org/10.1007/s11192-015-1556-z

Bongiovani, P., Gómez, N. D., y Miguel, S. (2012). Opiniones y hábitos de publicación en acceso abierto de los investigadores argentinos. Revista española de documentación cientifica, 35(3). https://doi.org/10.3989/redc.20 12.3.903

Bongiovani, P., Miguel, S., y Hernández-Pérez, T. (2017). Actitudes y percepciones de los evaluadores de la carrera científica en Argentina sobre la publicación en acceso abierto. Revista Española de Documentación Cientifica, 40(2), e171. https://doi.org/10.3989/redc.2017.2.1404

Budapest Open Access Initiative (2012). Diez años desde la Budapest Open Access Initiative: hacia lo abierto por defecto. Recuperado de http://www.budapestopenaccessinitiative.org/boai-10-translations/spanish

Budapest Open Access Initiative (2002). Declaration after the Open Society Institute meeting in Budapest. Budapest: Open Society Institute. Recuperado de http://www.soros.org/openaccess/read.shtml

Debat, H. y Babini, D. (2019). Plan S in Latin America: A precautionary note. PeerJ Preprints, 7, e27834v2. https:/ /doi.org/10.7287/peerj.preprints.27834v2

Fuchs, C., y Sandoval, M. (2013). The Diamond Model of Open Access Publishing: Why Policy Makers, Scholars, Universities, Libraries, Labour Unions and the Publishing World Need to Take Non-Commercial, Non-Profit Open Access Serious. tripleC, 13(2), 428-443. https://doi.org/10.31269/triplec.v11i2.502

International Seminar on Open Access (2006 [2005]) Declaración de Salvador sobre acceso abierto: la perspectiva del mundo en desarrollo. En D. Babini y J. Fraga (compiladores). Edición electrónica, bibliotecas virtuales y portales para las ciencias sociales en América Latina y El Caribe (pp. 237-238). Ciudad Autónoma de Buenos Aires: CLACSO. Recuperado de http://bibliotecavirtual.clacso.org.ar/ar/libros/secret/babini/Decla\%20Salva dor.pdf

Khoo, S. Y. S. (2019). Article processing charge hyperinflation and price insensitivity: an open access sequel to the serials crisis. LIBER Quarterly, 29(1), 1-18. http://doi.org/10.18352/lq.10280

Miguel, S., Gómez, N., y Bongiovani, P. (2012). Acceso abierto real y potencial a la producción científica de un país. El caso argentino. El profesional de la información, 21(2), 146-153. http://dx.doi.org/10.3145/epi.2012.mar.04

Pinfield, S., Salter, J., y Bath, P. A. (2016). The "total cost of publication" in a hybrid open\#access environment: Institutional approaches to funding journal article\#processing charges in combination with subscriptions. Journal of the Association for Information Science and Technology, 67(7), 1751-1766. https://doi.org/10.1002/ asi.23446

Pinfield, S., Salter, J., y Bath, P. A. (2017). A "Gold\#centric" implementation of open access: Hybrid journals, the "Total cost of publication," and policy development in the UK and beyond. Journal of the Association for Information Science and Technology, 68(9), 2248-2263. https://doi.org/10.1002/asi.23742

Piwowar, H., Priem, J., Larivière, V., Alperin, J. P., Matthias, L., Norlander, B., ... Haustein, S. (2018). The state of OA: A large-scale analysis of the prevalence and impact of Open Access articles. PeerJ, 6, e4375. https://doi.o $\mathrm{rg} / 10.7717 /$ peerj. 4375

Piwowar, H., Priem, J., y Orr, R. (2019). The Future of OA: A large-scale analysis projecting Open Access publication and readership. BioRxiv, 795310. https://doi.org/10.1101/795310

Science Europe (2019). Plan S: Making full and immediate Open Access a reality. Recuperado de https://www.scien ceeurope.org/coalition-s/

SCImago (2019). SJR — SCImago Journal \& Country Rank [Portal]. Recuperado de http://www.scimagojr.com 